\title{
Beitrag zur Kenntnis des Sklerödems der Erwachsenen.
}

\author{
Von \\ Stabsarzt Dr. Rissom, \\ Assistenten der Klinik.
}

Der Name Sklerödem ist von Soltmann für gewisse Krankheitszustände der Neugeborenen in die medizinische

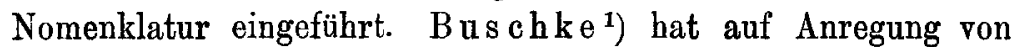
Lesser dieselbe Krankheitsbezeichnung für ein ähnliches Krankheitsbild beim Erwachsenen gewählt, wobei er betont, daß zwischen der Krankheit der Neugeborenen und dem von ihm als Sklerödem beschriebenen Krankheitsbild keine nachweisbaren Beziehungen beständen. P in k u s ${ }^{2}$ ) hat später 2 Fälle beschrieben, die große Ähnlichkeit mit dem B u s c h k e schen Sklerödem hatten. Im folgenden möchte ich über einen weiteren Fall von Sklerödem berichten und ihn mit den bisher veröffentlichten Fällen bezüglich der Symptomatologie und der Therapie vergleichen. Ich schicke eine kurze Skizze dieser Fälle voraus.

Fall 1. (Buschke l. c.) 46jähriger Mann, erkrankte im Anschluß von Influenza an Schwäche in den Gliedern, Steifigkeit der Finger, Starre in der Nackenregion. Die Starrheit breitete sich allmählich auf Gesicht, behaarten Kopf, Hals, Thorax, Abdomen und Rücken aus. Die Haut war stark geschwollen, bretthart, zeigte bei Fingerdruck keine Dellenbildung, war in Falten nicht zu erheben. An der Oberfläche sah sie stark gespannt, aber sonst normal aus, Man fühlte deutlich, daß es sich um ein in der Tiefe der Cutis, im subkutanen Gewebe und vielleicht auch in der Muskulatur liegendes Infiltrat handelte. Sensibilität frei. Gesichtsausdruck maskenartig, Atmung behindert, dergleichen Kopfbewegungen. Im Verlauf von 2 Jahren langsamer Rückgang der Erscheinungen. Die Haut wurde bis auf eine geringe Abnahme der Elastizität wieder normal. Nor die Wangenhaut blieb in einem elephantiasisartigen Zustand der Verhärtung. Keinerlei Atrophie, keine Pigmentveränderungen.

1) Buschke A. Über Sklerödem. Berl. klin. W. 1902. p. 955.

2) Pinkus F. Zwei Fälle von Sklerödem. Dermatologische Zeitschrift 1907. p. 425. 
Beim Eintauchen der Hände in kaltes Wasser vorübergehend ein Gefühl von Kriebeln und Taubsein in den Fingern.

Fall 2. (Pinkus l. c.) 48jähriger Mann bemerkt, ohne daB eine besondere Krankheit vorausging, Abnahme seiner Kräfte. Dabei Gefühl von Dickerwerden und Spannung in der Bauchhaut, zunehmende Steifheit, strammendes Gefühl in den Beinen, Kriebeln in Händen und Füßen. Von unten nach oben allmählich ansteigende Verhärtung der Haut. Haut allgemein verdichtet, besonders am Bauch und Rücken. An den erkrankten Stellen Hautoberfläche etwas heller, Fehlen der Cutis anserina beim Bestreichen, verminderte Schweißsekretion. Keine Sensibilitätsstörung. Die Haut macht den Findruck der pastösen Haut eines fetten Leicbnams. Bei Fingerdruck keine Dellenbildung. Haut nicht glänzend, sondern samtartig matt, wie gespannte weiche Haut. Algrenzung der kranken Haut von der gesunden nicht oberflächlich, sondern intrakutan, subkutan. An der Vorderfläche des rechten Oberschenkels von der Schenkelbeuge nach abwärts handtellergroße, scharf abgegrenzte, atrophische Hautstelle, die schon lange bestanden bat und traumatischen Ursprungs ist. Grobe Kraft der Arme und Beine vermindert. Nach Anwendung von Thiosinamininjektionen wesentliche Besserung. 40 Injektionen in Form des Fibrolysins dreimal wöchentlich 1 Dosis.

Mikroskopischer Befund eines excidierten Stückes der Rückenhaut: Elastisches Gewebe vollkommen normal; am kollagenen Gewebe weder Verdichtung noch Auflockerung, um die GefäBe keine Zeichen entzündlicher Infiltration. Vielleicht gewisse Raumbildung um die epithelialen Anhangsgebilde der Epidermis, etwa wie beim Ödem. Gefäße von zahlreichen Mastzellen eingescheidet, reichlich Zellen voll braungelben Pigments, teils um die Gefäße, teils im Papillarkörper verstreut.

Fall 3. (Pinkus 1. c.) 58jähriger Mann. Allmähliche Krankheitsentwicklung mit zunebmender Schwäche und Schwerfälligkeit, Gewichtsanstieg, zunehmender Hautspannung. Haut dick, gespannt, schwer faltbar, oberflächlich weich, in "der Tiefe hart wie fester Speck. Die Verhärtung erstreckt sich über den ganzen Brustkorb, Bauch und Rücken, Oberarme und obere Drittel der Oberschenkel. Klagen über Schmerzen und unangenehme Sensationen in den Füßen, Unterschenkeln und Oberschenkeln. Grobe Kraft elwas herabgesetzt. Gesicht rot, dick, zeigt grämlichen Ausdruck. Schildrüse klein, hart.

Ich komme nun zu der Schilderung des ron mir in oben bezeichneter Klinik beobachteten Krankheitsfalles.

Fall 4. Vorgeschichte. P. M., Weichensteller, 45 Jahre alt, erkrankte am 8. Januar 1906 unter infuenzaartigen Erscheinungen. Gleichzeitig trat eine ekzemartige Entzündung der Haut des Halses und in der Umgebung der Brustwarzen auf. Es bildeten sich Pustelchen, die aufbrachen und eiterige Flüssigkeit sezernierten. Diese Entzündung ging in etwa 8 Tagen zurück. Es entwickelte sich aber nun eine rasch über Gesicht, Hals, Schultern und Thorax sich ausbreitende Hautschwellung. Die Haut fühite sich brettartig hart an, hatte eine rotblaue Farbe. Die 
Augenlider waren geschwollen, die Augen sahen trübe aus. (Der Ausdruck des Kranken lautete „blind“.) Das Gesicht war durch die Schwellung erheblich entstellt, ähnelte dem eines Trunkenen. Die Bewegung der Arme war durch die Hautschwellung behindert. Die Arme waren in rechtwinkeliger Beugestellung, der Kopf in nach vorn gebeugter Haltung fixiert; die Atmung war durch die brettartige Haut behindert. Das Gefühl war in der geschwollenen Haut herabgesetzt. In beiden Händen soll ein Gefühl von Kriebeln bestanden haben. Nach 8wöchiger Behandlung besserte der Zustand sich soweit, daß der Kranke wieder arbeiten konnte. Dabei beobachtete der Kranke, daß sein Zustand in der Kälte und nach längerer Ruhe sich verschlechterte, während bei Erwärmung und besonders bei körperlicher Arbeit die Geschmeidigkeit der Haut wieder zunahm. In den folgenden zwei Jahren änderte sich der Zustand wenig. Auf Perioden der Besserung folgten Zeiten der Verschlechterung, letztere besonders während der kalten Jahreszeit. Die grobe Kraft des Körpers, insbesondere der Arme soll im Laufe der Zeit abgenommen haben. Fine 6wöchige klinische Behandlung in Kiel brachte keine Besserung, Die Behandlung in der Kieler Klinik bestand angeblich in Schwitzprozeduren, Galvanisation und Arsendarreichung. Seitens des behandelnden Arztes ${ }^{1}$ ) wurden längere Zeit Thyreoidintabletten gegeben, jedoch ohne Erfolg, ferner längere Zeit Arsen.

A u fnahmebefund 4./I. 1908. Mittelgroßer Mann, in ausreichendem Ernäbrungszustand. An den inneren Organen keine Krankheitszeichen. Der Urin ist frei von Eiweiß und Zucker. Körperwärme normal.

Die Gesichtsfarbe ist gelbrötlich. Die Haut des Körpers zeigt einen gelblichen, ins wachsfarbene gehenden Farbenton. Im Gesicht fällt die Starrheit der Züge auf, sonst sieht man weder im Gesicht noch am Stamm eine Veränderung der oberflächlichen Hautschichten, insbesondere sind keine atrophischen Stellen und keine Pigmentanomalien zu finden. Beim Betasten fällt am ganzen Körper mit Ausschluß der Hände und Füße eine eigentümliche Steifigkeit der Haut auf. Dem Gefühl nach hat diese Steifigkeit ihre Ursache in einer Verhärtung der tieferen Cutisschichten und der Subcutis. Im Gesicht läßt sich die Haut nicht in den normalen Falten abheben, den Jochbögen liegt sie straff.an und ist kaum verschieblich. Am Nacken ist die Härte der Haut noch hochgradiger. Hier läßt sich die Haut weder verschieben noch abheben. Etwas weicher ist sie an der Vorderfläche des Halses. Am Rumpf, sowobl am Rücken wie an Brust und Bauch läßt sich trotz mäßiger Fettentwicklung die Haut nur in ganz flachen Falten emporheben. Sie ist nur in ganz geringem Maße auf der Unterlage verschieblich, fühlt sich an wie mit hartem Paraffin durchtränkt und umschließt den Körper als starrer Panzer. In geringerem $\mathrm{Maße}$ ist die Haut der Oberschenkel und des oberen Drittels der Unterschenkel ergriffen. Die Haut der Oberarme ist

1) Dr. Eg g e rs, Marne i. H., für die von ihm freundlichst erteilte Auskunft über die Vorgeschichte sage ich an dieser Stelle meinen besten Dank. 
besonders an den Streckseiten verhärtet und verkürzt und umschließt die Muskulatur als straffer Schlauch. Die Haut der Unterarme ist ziemlich weich, macht aber auch den Eindruck, als sei sie zu eng. An den Händen und Füßen ist die Haut normal. Die verbärteten Hautteile gehen ohne scharfe Grenze allmählich in die gesunden Teile über. An beiden Unterschenkeln finden sich tiefe Schnürfurchen von den Strumpfbändern, sonst bei Fingerdruck keine Dellenbildung.

Die Mimik des Gesichts ist auffallend träge. Beim Stirnrunzeln zeigen sich nur einige flache Falten auf der Stirn, beim Heben der Mundwinkel vertieft sich die Nasolabialfalte nur wenig. Durch die Rigidität der Nackenhant sind die Kopfbewegungen nach den Seiteu und nach vorne behindert. Der Kranke vermag das Kinn nicht der Brust völlig zu nähern. Auch die Beweglichkeit der Arme ist gestört. Der Kranke vermag die erhobenen Hände nach hinten nur bis zum Hinterkopf zu bringen, erreicht also nicht die Nackengegend und vermag z. B. nicht, sich den Kragen hinten am Hemd anzuknöpfen. Bei tiefer Atmung macht sich ein Gefühl von Beengung in der Brust geltend. Beim Schlucken verspürt der Kranke ein Gefühl von Steifigkeit im Schlunde. Objektiv finden sich im Rachen keine Veränderungen.

Die Muskulatur der Arme ist nur mäBig kräftig entwickelt.

Die Sensibilität zeigt keine Störungen. Das Gefübl für zarte Berührung, die Unterscheidung von warm und kalt, von spitz und stumpf ist überall vorhanden.

Bebandlung: Salol 100 zweimal täglich. Morgens: warmes Bad. Abends: Allgemeine Körpermassage. Vom 27./I. ab Fibrolysininjektionen.

Krankheitsverlauf. In den nächsten Tagen traten am Rumpf zahlreiche, ringförmige, leicht infiltrierte, rote Eftloreszenzen - Urticaria rubra auf, die nach Regelung der Diät in zwei Tagen wieder verschwanden.

Unter dem Einfluß der eingeschlagenen Behandlung machte sich bald eine Besserung des Zustandes der Haut bemerkbar, die sowobl subjektiv wie objektiv zur Geltung kam.

Vom 27./I. 1908 erhielt der Kranke jeden 2. Tag $23 g$ Fibrolysin $=0.2 g$ Thiosinamin intramuskulär. Die Einspritzungen wurden gut vertragen. Der Puls bl:eb regelmäßig 62 -72. Der Urin frei von Eiweiß. Die Besserung machte weitere Fortschritte. Die Haut wurde besonders am Nacken und Hals, dann aber auch am Stamm erheblich weicher.

Nach 20 Iajektionen in 40 Tagen konnte der Kranke am 4//III. 1908 in recht befriedigendem Zustande die Klinik verlassen. Die Beweglichkeit der Gesichtszüge war ziemlich normal, auch waren die Kopf bewegungen frei, doch war die Haut am ganzen Rumpf noch deutlich verhärtet.

Am 6./VI. 1908 stellte sich der Kranke wieder vor. Er gab an, bald nach der Entlassung aus der Klinik von Herzbeschwerden, bestehend in Atemnot und lebhaftem Herzklopfen befallen zu sein. Nach einigen Tagen Bettruhe seien die Beschwerden wieder zurückgegangen. Im übrigen habe sich der Zustand seiner Haut weiter gebessert.

Objektiv fanden sich am 6./VI. 1908 am Herzen keinerlei nachweisbare Veränderungen. Herzdämpfung nicht verbreitert, Herztöne rein, 
Puls regelmäßig 72. Urin frei von Eiweiß und Zucker. Der Allgemeinzustand des Kranken war ein günstiger. Er sah gesund aus und war gut genährt. Kopfbewegungen nach den Seiten und nach vorn und hinten völlig unbehindert; keinerlei Beengung bei der Atmung. Die frühere Bebinderung der Armbewegungen ist völlig verschwunden. Der Kranke erreicht jetzt mühelos mit beiden Händen die Gegend zwischen den Schulterblättern. Die Haut des Gesichts ist kaum noch verhårtet. Die Gesichtsbewegungen sind ungestört. Die Haut des Stammes ist noch etwas derber als der Norm entspricht, aber gut verschieblich und überall in mäßig dicken Falten, die dem Panniculus adiposus entsprechen, abhebbar. Besonders deutlich ist die Besserung der Arme. Sowohl an den Vorderarmen wie an den Oberarmen ist die Hant normal verschieblich und zeigt die Weichheit und Turgeszenz der gesunden Haut. Die Haut des Nackens ist fast normal.

Der Kranke ist mit seinem Zustand sehr zufrieden. Leider wird seine Arbeitsfähigkeit durch eine inzwischen eingetretene hartnäckige Is c hi as beeinträchtigt.

Mikroskopischer Befund. Untersucht wurde ein am 23./I. 1908 excidiertes Hautstückchen aus der Lendengegend. Die Epidermis zeigt regelrechte Entwicklung der einzelnen Schichten. Im Corium ist das elastische Fasernetz in normaler Weise ausgebildet. Die Dicke des Coriums erscheint normal; an den kollagenen Fasern ist weder in der Form noch in der Dichtigkeit etwas besonderes wahrzunehmen. Keine entzündlichen Infiltrate um die Blutgefäße. Das Unterhautzellgewebe, das bis auf die Fascie ausgelöst wurde, zeigt reichliche Fetteinlagerung. Etwa in der Mitte derselben ist ein parallel der Cutis verlaufender derber Bindegewebszug eingelagert, der in allen Schnitten wiederkehrt und einer ins Unterhautzellgewebe eingelagerten fibrösen Platte entspricht. Auch in diesem Bindegewebszug finden sich keine entzündlichen Elemente.

Symptomatologie. Die Analogien sowohl in Decursus als in den Symptomen der zusammengestellten Fälle sind deutlich zu erkennen. Bezüglich des 3. Falles ( $\mathrm{inkus)} \mathrm{fehlen} \mathrm{leider}$ Daten über den weiteren Verlauf. Pinkus selbst betrachtet es als unsicher, ob dieser Fall dem Sklerödem zuzurechnen sei, oder ob derselbe den Fällen von Adiposis dolorosa näher stände. Die drei anderen Fälle gehören sichei zusammen. Der Beginn des Leidens zeigt graduelle Differenzen. Während Fall 2 einen schleichenden Beginn zeigt, ist in Fall 1 das Einsetzen des Leidens schon ziemlich plötzlich, und wird von $B$ uschke als 
subakut bezeichnet. In unserem Fall kann man von einem akuten Einsetzen des Sklerödems reden. In wenigen Tagen entwickelte sich das Krankheitsbild zur vollen Höhe. Die Versteifung der Haut wurde in 8 Tagen so hochgradig, daß die Arme in rechtwinkeliger Beugestellung fixiert waren. Im Fall 1 entstand die Krankheit im Anschluß an Influenza. Auch in unserem Fall schlob sich die Krankheit an influenzaartige Erscheinungen an. Bei Fall 2 und 3 trat das Hautleiden allmählich ein, ohne daß eine fieberhafte Affektion vorausgegangen war. Als wesentlichstes Symptom findet sich in allen Fällen eine eigenartige Verhärtung und Versteifung der Haut. Die Haut fühlt sich an wie die "steif gewordene Haut einer Leiche" oder wie "mit hartem Paraffin durchtränkt". Der Sitz dieser Verhärtung der Haut wird dem Gefühl nach übereinstimmend in die tieferen Schichten der Cutis und in die Subcutis verlegt. Die obersten Schichten der Haut wurden sowohl nach dem Gefühl als nach dem Aspekt stets normal gefunden. Die Haut machte den Eindruck praller ödematöser Durchtränkung, doch ließ sich bei Fingerdruck keine Dellenbildung erzielen. Atrophische Veränderungen an der Hautoberfläche oder Veränderungen in der Pigmentverteilung im Verlauf des Krankheitsprozesses wurden in keinem Falle gefunden. Pinkus fand in Fall 2 ain rechten Oberschenkel eine umschriebene handtellergroße Hautatrophie. $\mathrm{Er}$ hält es jedoch fïr unwahrscheinlich, daß diese Atrophie mit dem Sklerödem in ätiologischem $\mathrm{Zu}$ sammenhang stände, nimmt vielmehr für die Entstehung derselben ein Trauma an. Lokalisiert war die Hautveränderung vorwiegend am Rumpf, Nacken, Gesicht und den oberen Abschnitten der Extremitäten, während Hände und Füße abgeseben von sensorischen Störungen frei blieben. Die Folge der Hautveränderung war eine erhebliche Behinderung der Beweglichkeit. Kopfbewegungen, Atmung und Armbewegungen waren in ihren Exkursionen verringert. Das Gesicht war maskenartig; zeigte mürrischen, grämlichen Ausdruck und verlangsamte Mimik.

Die Berührungsempfindlichkeit war in allen Fällen ungestört. Buschke sowohl wie Pinkus heben hervor, dab ihre Kranken an Parästhesien, Kriebeln in der erkrankten Haut, besonders in den Extremitäten litten. Auch dieses Symptom findet 
sich in unserem Fall. Als bemerkenswert tritt in diesem Fall die Empfindlichkeit der Haut gegen Temperatureinflüsse hinzu, Verschlimmerung des Zustandes bei Abkühlung, Besserung bei Erwärmung und körperlicher Arbeit. Auch im Fall 1 findet sich, wenn auch weniger ausgesprochen, eine vermehrte Empfindlich keit gegen Kälteeinwirkung. Der Kranke bekam, wenn er die Hände in kaltes Wasser steckte, rorïbergehend ein Gefühl von Kriebeln, Taubsein, Eingeschlafensein in den Fingern.

Buschke und Pinkus fanden die Schweißsekretion an den erkrankten Hautteilen vermindert. Bei unserem Fall ist mir das nicht aufgefallen.

Über den Verlauf des Leidens liegen Beobachtungen in Fall 1,2 und 4 vor. Gemeinsam ist diesen Fällen eine entschiedene Tendenz zur Heilung. Sowobl Buschke wie Pinkus beobachteten Ausgang in fast rollständige Heilung. Auch der von mir beobachtete Fall ließ sich günstig beeinflussen und scheint der Heilung entgegen zu gehen. Wichtig ist, daß der Rückgang der Hautschwellung in keinem Fall von Atrophie der Haut begleitet war.

Diagnostisch erhebt sich die Frage, ob es berechtigt ist, die geschilderten Fälle unter dem Namen Sklerödem von der Sklerodermie abzugrenzen. Blaschko ${ }^{1}$ ) ist der Ansicht, daß diese Fälle der Sklerodermie einzureihen seien. Er nimmt an, daß es sich um einen der Sklerodermie identischen Krankeitsprozeß handelt, der sich nur durch die Lokalisation in den tieferen Hautschichten von der typischen Sklerodermie unterscheidet. Demgegenüber ist geltend zu machen, daß der ganze Decursus morbi doch in den geschilderten Fällen erheblich von der Sklerodermie abweicht. Das plötzliche Einsetzen der Ödeme, ihre schnelle Ausbreitung, ihr tiefer Sitz, die Neigung des Krankheitsprozesses zur Heilung ohne sekundäre Atrophie der befallenen Hautteile, ohne Pigmentreränderungen, das alles sind Momente, die der Sklerodermie fremd sind und die darauf hinweisen, daß es sich hier um einen hinreichend charakterisierten, von der Sklerodermie versehiedenen Krankheitsprozeß sui generis handelt.

Was nun das Wesen der Krankheit an sich angeht, so läßt sich zur Zeit hierüber nur wenig sagen. Die pathologisch

1) Blaschko, Verhandlungen der Berl, derm. Gesellsch. 1900. Derm. Zeitschrift. 1900, p. 881. 
anatomische Untersuchung beschränkt sich auf mikroskopische Untersuchung excidierter Hautstückchen. Das Resultat dieser Untersuchungen, die im Fall 2 von Pinkus, in unserem Falle von mir vorgenommen warden, jst ziemlich dürftig. Entzündungserscheinungen waren nicht nachzuweisen, auch fand sich weder in der Schichtung noch in der Struktur der Haut etwas von der Norm abweichendes. Auch an den Blut- und Lymphgefäßen waren weder Entzündungs- noch Staungserscheinungen zu entdecken. Dennoch macht es der klinische Befund wahrscheinlich, daß die Ursache der Hautrersteifung in einer Lymphstockung zu suchen ist. Wie diese Lymphstockung zu stande kommt, ist schwer $\mathrm{zu}$ sagen. Auffallend ist namentlich in unserem Fall die hochgradige Beeinflußbarkeit des Krankheitszustandes durch Temperaturunterschiede, Zunahme der Hautverhärtung bei Abkühlung, Besserung bei Erwärmung. Es wäre möglich, daß es sich hierbei um eine krankhafte Störung der Innervierung der Hautgefäße handelt, die primär bestehend mit dem Ödem in ätiologischem Zusammenhang stehen könnte. Aber das läßt sich nicht beweisen. Ebenso gut kann es sich um die an sich normale Folge eines Temperaturreizes handeln, die sich infolge des pathologischen Zustandes der Haut in pathologischer Weise äußert. Schon Buschke spricht die Vermutung aus, dah das primäre eine Erkrankung des Lymphgefäßsystems sei. Er stützt sich hierbei auf Senator, der denselben Fall vor ihm beobachtete. S enator hält es für möglich, daß es sich um eine Erkrankung der Hautlymphgefäße infolge Einwirkung des Influenzatoxins handle. Wie Eingangs erwähnt, waren in diesem Fall typische Influenzaerscheinungen dem Hautleiden vorausgegangen. Auch in unserem Fall schloß sich das Hautleiden an influenzaartige Allgemeinerscheinungeu an. Nach Mitteilung des behandelnden Arztes handelte es sich aber weniger um eine echte Influenza als um fieberhafte Allgemeinsymptome unbestimmter Natur. Ich halte es daher für möglich, daß es sich in unserem Fall nicht um Influenza, sondern um fieberhafte Prodrome, resp. Anfangssymptome des akut einsetzenden Sklerödems gehandelt hat. Ich möchte daher mindestens in unserem Fall das Sklerödem nicht als Folge der Influenza ansehen. 
Die Prognose des Sklerödems ergibt sich aus den oben gemachten Mitteilungen über den Verlauf. Nach den bisherigen Beobachtungen darf sie in Gegensatz zur Sklerodermie als nicht ungünstig bezeichnet werden.

Der Therapie bietet das Sklerödem ein dankbares Feld. In erster Linie haben sich medicomechanische Prozeduren als günstig erwiesen. Insbesondere verdient die allgemeine Körpermassage ausgiebigste Verwendung. Uns gelang es durch die Massage in wenigen Tagen eine erhebliche Besserung zu erzielen. Als Gleitmittel wurde Olivenöl benützt. Daneben erhielt unser Kranker Salol. Pinkus sah guten Erfolg ron intramuskulären Fibrolysineinspritzungen. Er applizierte 40 Spritzen. Unser Kranker erhielt 20 Injektionen zu $2 \cdot 3 g$ Fibrolysin gleich $0.2 g$ Thiosinamin p. d. Das Thiosinamin wurde zuerst von H. v. Hebra ${ }^{\text {) }}$ in die Therapie der Sklerodermie eingeführt. Die von Hebra beobachtete günstige Wirkung wurde von Juliusberg ${ }^{2}$ ) bestätigt. Auch uns hat das Fibrolysin gute Dienste geleistet, insbesondere glauben wir das Fortschreiten der Besserung nach Abschluß der Behandlung auf Rechnung des Fibrolysins setzen zu dürfen. Eine unangenehme Eigenschaft des Thiosinamins ist es, daß es bisweilen toxisch wirkt. Bei unserem Kranken traten nach Abschluß der Fibrolysinkur Herzstörungen hervor, die in Herzklopfen und hochgradiger Atemnot bestanden und den Kranken für mehrere Tage aufs Lager warfen. Dauernden Schaden hat der Kranke jedoch nicht davon getragen. Das gewöhnliche Bild der Thiosinaminintoxikation sind influenzaartige Erscheinungen mit mäßigem, manchmal hohem Fieber und sehr erheblicher allgemeiner Körperschwäche. Wie heimtückisch das Thiosinamin unter Umständen wirkt, zeigt ein von Gross $\mathrm{e}^{3}$ ) publizierter Fall. Sein Kranker, der sich șchon nach der ersten Fibrolysininjektion angegriffen fühlte, bekam nach der 6. Spritze schwere Intoxikationserscheinungen. Herzschwäche, hohes Fieber, Anurie,

1) H. v. Hebra. Drei Fälle von Sklerodermie, behandelt mit Thiosinamin. Arch. f. Derm. 1899. Bd. XLVIII. p. 120.

y) Juliusberg. Über Wirkung etc. des Thiosinamins. Deutsche medizinische Wochenschrift. 1903. p. 591.

3) Grosse. Ein Fall von Vergiftung nach Gebrauch von Thiosinamin. Münch. med. W. 1908. p. 910. 
Erbrechen, hochgradiger Verfall der körperlichen und geistigen Kräfte waren die Hauptmerkmale des bedrohlichen Zustandes, aus dem der Kranke sich erst im Laufe von 4 Wochen erholte. Solche Fälle mahnen, das Thiosinamin nur unter zuverlässiger Kontrolle von Puls und Körperwärme zu verwenden und dasselbe nach Möglichkeit der stationären Behandlung vorzubehalten. Zum Schluß sei noch bemerkt, daß ein Versuch des behandelnden Arztes, den Krankheitsprozeß durch Darreichung von Thyreoidintabletten zu beeinflussen, nach schriftlicher Mitteilung des Arztes keinen Erfolg hatte.

\section{Zusammenfassung,}

1. Das Hauptsymptom des Sklerödems ist ein ausgebreitetes tiefsitzendes Ödem der Haut, das eine Versteifung und Verhärtung der Haut in ihren tieferen Schichten zur Folge hat. Die obersten Hautschichten bleiben vollständig normal.

2. Das Sklerödem setzt entweder akut oder subakutmit vorausgehenden Fiebererscheinungen oder von vornherein mit chronischem Verlauf ein. Die Rückbildung des Sklerödems erfolgt ohne Pigmentanhäufung und ohne Atrophie an den befallenen Hautstellen.

3. Das Sklerödem beruht wahrscheinlich auf einer Lymphstockung infolge Erkrankung der HautlymphgefäBe. Die mikroskopische Untersuchung excidierter Hautstückchen hat bisher nichts ergeben, was die klinischen Symptome erklärt.

4. Für die Behandlung des Sklerödemsempfiehlt sich neben allgemeiner Körpermassage die Darreichung von intramuskulären Thiosinamininjektionen (Fibrolysin). Thyreoidbehandlung erwies sich als wirkungslos. 